\title{
Optimized regimens of combined medications for the treatment of major depressive disorder: a double-blind, randomized-controlled trial
}

This article was published in the following Dove Press journal: Neuropsychiatric Disease and Treatment

\section{Zoë Zuilhof \\ Sandhaya Norris \\ Claude Blondeau \\ Pierre Tessier \\ Pierre Blier}

Department of Psychiatry, University of Ottawa, The Royal Ottawa Institute of Mental Health Research, Ottawa, ON, Canada
Correspondence: Pierre Blier The Royal Ottawa Institute of Mental Health, I I 45 Carling Avenue, Ottawa, ON, Canada

Email pierre.blier@theroyal.ca
Introduction: This study investigated if optimized dose regimens of escitalopram and bupropion combination from treatment initiation can be superior to either drug alone in speed of onset, remission rate, and maintenance of therapeutic efficacy.

Methods: Patients from a single site $(\mathrm{N}=85)$ within a larger double-blind 12-week trial $(\mathrm{N}=245)$ showed a lower dropout rate (14\% vs $40 \%)$ and used higher doses; therefore, this cohort was analyzed separately. Uniquely at this single site, after 12 weeks, non-remitters on a single drug received the other one in addition and combination non-remitters underwent a switch of escitalopram for duloxetine for a 6-week period. Escitalopram could be given up to $40 \mathrm{mg}$ /day and bupropion up to $450 \mathrm{mg} /$ day. A 6-month prolongation was then implemented in remitters, maintaining the double-blind design throughout. Remission was defined as $\leq 7$ on the 17 -item Hamilton Rating Scale for Depression, as in the initial publication.

Results: At week 2, combination treatment was superior in remission rate (5/28) compared with both bupropion ( $0 / 26)$ and escitalopram monotherapies $(0 / 31 ; P=0.03$ and $P=0.02$, respectively). The week 12 remission rate of combination treatment showed a higher rate $(15 / 28)$ relative to bupropion monotherapy $(7 / 26 ; P=0.04)$, but not statistically different from escitalopram monotherapy $(11 / 31 ; P=0.13)$. The 6 -week augmentation produced remission in $7 / 21$ monotherapy non-remitters and $0 / 6$ in the switch group $(P=0.13)$. Remission was sustained in 28/31 patients enrolled in the 6-month maintenance.

Conclusion: These results suggest that combination of escitalopram and bupropion from treatment initiation is superior to either monotherapy in speed of onset. The addition of a second drug in non-remitters can lead to additional remissions, as shown with other combinations of medications. Treatment prolongation using optimized regimens leads to low relapse rates.

Keywords: antidepressant, escitalopram, bupropion, action onset, augmentation, prolongation

\section{Introduction}

Despite the high prevalence of major depressive disorder (MDD), ${ }^{1}$ current available treatment options are still insufficient, resulting in persistent disabling symptoms, lower work productivity, higher health care costs and suicides. ${ }^{2}$ Both pharmacological and psychotherapeutic treatments generally produce a slow onset of action and low remission rates. The first-line medications, like selective serotonin reuptake inhibitors (SSRIs), may take 2 weeks before any improvement occurs and after 6 weeks, only about one-third of patients attain remission, one-third shows partial response, and one-third fails to respond at all. ${ }^{3-5}$ Nonresponders to at least two adequate pharmacological trials with appropriate dosing and duration may be classified under the term treatment-resistant depression (TRD). ${ }^{6}$ 
Another problem with the treatment of MDD is that, although there exists a reliable predictor of nonresponse (ie, the lack of a $20 \%$ improvement after 2 weeks of treatment with a single drug), predictors of a positive response are yet lacking. ${ }^{7,8}$ Nevertheless, specific medications can be selected in first intent to improve rapidly certain symptom domains (ie, mirtazapine or agomelatine for insomnia). However, following treatment failures many patients end up on a combination of more than one medication, based on different mechanisms of action. ${ }^{9}, 10$ Hence, medication augmentation is a common practice for treatment-resistant patients. ${ }^{11,12}$ Unfortunately, the longer the delay in achieving remission in these patients, the worse is their depression prognosis and the harder achieving remission becomes. ${ }^{13,14}$ Vice versa, early onset of efficacy of antidepressant therapy is vital, considering it is associated with a more stable response, a higher remission rate, and less recurrence of depression than late onset. ${ }^{14}$ Thus, starting with more than one medication from treatment initiation may be a worthwhile approach.

Four randomized, double-blind, placebo-controlled studies using optimized dose regimens support the potential superiority of a combination strategy from treatment initiation. ${ }^{15-18}$ Maes et al initiated the administration of the SSRI fluoxetine with a placebo or with pindolol or the $\alpha_{2}$-adrenergic, $5-\mathrm{HT}_{2 \mathrm{~A}}$ and $5-\mathrm{HT}_{2 \mathrm{C}}$ antagonist mianserin to treat MDD in $31 \mathrm{TRD}$ patients. ${ }^{15}$ The SSRI-mianserin approach outperformed the SSRI alone in terms of overall response in the first week and in end-of-study, week 5, the fluoxetine monotherapy resulted in only a $9 \%$ response rate compared with $60 \%$ in both the SSRI-pindolol and the SSRI-mianserin arms. Nelson et al assessed the efficacy of an SSRI, a norepinephrine (NE) reuptake inhibitor, and their combination in 39 hospitalized MDD patients. ${ }^{16}$ After 6 weeks, the SSRI fluoxetine and the NE reuptake inhibitor desipramine had yielded a superior remission rate in combination relative to either medication alone: $53 \%$ vs $7 \%$ and $0 \%$, respectively. Likewise, combination therapy using mirtazapine and the SSRI paroxetine was compared to either drug alone in $61 \mathrm{MDD}$ patients, and week 6 remission rates for the combination, mirtazapine or paroxetine monotherapy showed $53 \%$ vs $19 \%$ and $26 \%$, respectively. ${ }^{17}$ Moreover, half of the monotherapy nonresponders remitted after a 2-week augmentation with the other drug. In $105 \mathrm{MDD}$ patients, various combinations with mirtazapine (ie, bupropion, fluoxetine, and venlafaxine) all attained superior remission rates compared with fluoxetine alone: $25 \%$ vs $46 \%-58 \% .{ }^{18}$ Confirming the efficacy of the combinations, a double-blind discontinuation of one agent in the combination responders resulted in $40 \%$ early relapse.
In contrast to these findings, two larger trials found different results but they were subjected to methodological limitations. A large placebo-controlled, but single-blind, trial known as the Combining Medications to Enhance Depression Outcomes (CO-MED; $\mathrm{n}=668$ ) study compared two combinations to escitalopram monotherapy after an initial 1-week open-label period on one medication, and showed a similar remission rate of $40 \%$ in all groups. ${ }^{19}$ Importantly, the investigators were never blinded to the treatment of the patients and the dosing regimen ended up being suboptimal in the two combination groups. The mean exit dose of mirtazapine was subtherapeutic (ie, $22 \mathrm{mg} /$ day on average), that of venlafaxine was $<200 \mathrm{mg} /$ day, and that of escitalopram was lower in the combination group than in the monotherapy group. Therefore, a four-center, double-blind, randomized, placebo-controlled study investigated again the escitalopram and bupropion combination therapy compared to either drug alone using optimized dose regimens. ${ }^{20}$ Doses above the maximal recommended dose of escitalopram of $20 \mathrm{mg}$ /day (ie, 30 and $40 \mathrm{mg} /$ day) and up to a maximal dose of bupropion of $450 \mathrm{mg} /$ day were titrated weekly. Although this randomized trial in 245 MDD patients put into evidence a significant superiority of higher doses of escitalopram (on average $30 \mathrm{mg}$ /day) over bupropion at the end of 12 weeks of initial treatment (remission rates of $52 \%$ vs $34 \%$, respectively), the combination was not superior to either drug alone. It is noteworthy that a primary outcome measure of remission at week 2 showed that the combined treatment achieved statistically significant superiority over bupropion, but not quite vs escitalopram monotherapy.

There were, however, significant differences in dose levels and dropout rates between the four clinical units involved in this study. Nearly all patients at a single site, the Royal Ottawa Institute of Mental Health Research (IMHR) unit, reached the maximal possible doses, yet the dropout rate was much lower ( $14 \%$ vs $34 \%-40 \%$ in the other locations). Consequently, these two observations on their own justified an analysis of the results obtained at this single site. Current report thus examined this IMHR subsample within a larger multisite trial. In addition, uniquely at this site, a subsequent double-blind, 6-week augmentation or switch strategy was carried out in 27 patients who did not achieve remission. Those on a single medication kept their medication regimen and the other drug was added, whereas the combination group underwent a switch of the escitalopram for duloxetine. The hypotheses were that in this subsample, 1) the onset of remission will be faster in combination therapy relative to both bupropion and escitalopram monotherapies as measured by 
response and/or remission in weeks 1 and 2;2) the remission rate after 12 weeks will be greater in combination therapy than in both monotherapies as measured by week 12 remission rate; 3 ) adding the other medication in monotherapy, nonresponders will produce additional remitters as measured by augmentation week 6 remission; and 4) combination therapy will sustain remission better than monotherapies as measured by month 6 remission rates.

\section{Materials and methods Subjects}

Patient evaluation was performed using the Structured Clinical Interview for Diagnostic and Statistical Manual of Mental Disorders, Fourth Edition, Text Revision (DSM-IV-TR) Diagnoses. ${ }^{20}$ Alongside the MDD diagnosis, patients had to show $\geq 22$ score on the Montgomery-Asberg Depression Rating Scale (MADRS) for randomization. ${ }^{20}$ Exclusion criteria were comorbid psychosis or another axis I disorder other than MDD considered primary, as well as bipolar disorder or an eating disorder, a history of seizures or an abnormal electrocardiogram. Patients taking concomitant medications for an unstable physical condition or receiving other psychoactive drugs were not eligible, whereas patients using medications to maintain a stable physical condition, eg, a controlled blood pressure, were allowed. ${ }^{20}$ All patients provided written informed consent before starting their study participation. After general health was confirmed by physical examination and laboratory tests, which included a drug screening, they were randomized to either bupropion or escitalopram monotherapy or treatment with the combination of both. In the augmentation phase, nonremitters on initial monotherapy kept their current regimen and the other drug was added, whereas non-remitters on initial combination treatment underwent a switch of escitalopram for duloxetine in addition to continued administration of bupropion, with weekly titration as tolerated.

\section{Dose regimen}

Four different dose levels were used, based on tolerability and with the patient's agreement, the level was maintained or increased by one level per week. In the combination treatment, the dose levels were the same as each drug in monotherapy. Level 1 was $150 \mathrm{mg}$ bupropion and/or $10 \mathrm{mg}$ escitalopram. In week 2, they could start on level 2, ie, $300 \mathrm{mg}$ bupropion and/or $20 \mathrm{mg}$ escitalopram. In week 3, this could be upgraded to level 3, $300 \mathrm{mg}$ bupropion and/or $30 \mathrm{mg}$ escitalopram. From week 4 onward, dose levels could go up to level 4, corresponding to $450 \mathrm{mg}$ bupropion and/or $40 \mathrm{mg}$ escitalopram..$^{20}$ Level 1 of duloxetine was $60 \mathrm{mg} /$ day, levels 2 and 3 were $90 \mathrm{mg} /$ day, and level 4 was $120 \mathrm{mg} /$ day. Randomization was done by computer. The medications and the placebos were appearing identical, they were provided in sealed dispensing cards, and the number of tablets to be taken daily in the morning with food was the same at all levels to prevent patients from knowing which group they had been assigned to. The raters were blind to the treatment groups.

\section{Endpoints}

Throughout the study, the primary outcome of efficacy was assessed weekly on the 17-item Hamilton Rating Scale for Depression (HAM-D17), as in the overall report. ${ }^{20}$ The MADRS was used for the study entry screening and assessed secondarily at weeks 2 and 12 and at the end of the 6-week augmentation. ${ }^{20}$ Remission on the HAM-D17 was defined by a score of $\leq 7$ and on the MADRS as $\leq 10$. $^{20}$

\section{Data analysis}

Overall sample size determination for the first phase of the trial was outlined in Stewart et al. ${ }^{20}$ To correct for missing data of dropouts and for missed study visits of completers, all data were analyzed in a last observation carried forward (LOCF) manner. All values are represented as mean \pm standard deviation. Multivariate analyses of variance (MANOVAs) were used for change score comparisons as well as MANOVA or ANOVA for dose-level examinations, and remission rates were analyzed by chi-squared tests or Fisher's exact tests, in case a remission rate of zero was included. All statistical analyses were tested against a significance level of $\alpha=0.05$ and performed in SPSS version 22.0. ${ }^{21}$ MANOVA post-hoc least significant difference (LSD) comparisons were used, without correction for multiple testing, justified by the modest sample size and loss of power when using MANOVA for a repeated measures design.

All three phases of the study were approved by the Royal Ottawa Health Care group research ethics board and funded by NIMH 1R01 MH077285-01A2. Health Canada Clinical Trial number: 120040 .

\section{Results}

Of the screened patients for the study, not all met the inclusion criteria and some fell in the exclusion criteria at the time of randomization, resulting in a lower but substantial eligible number (Figure 1). By exemption, two patients had to be excluded because of errors made by pharmacy. There were no statistical differences in age, sex, and marital status among the three groups, as was the case in the larger sample (Table 1). ${ }^{20}$ Dropout rates were $19 \%$ in bupropion 


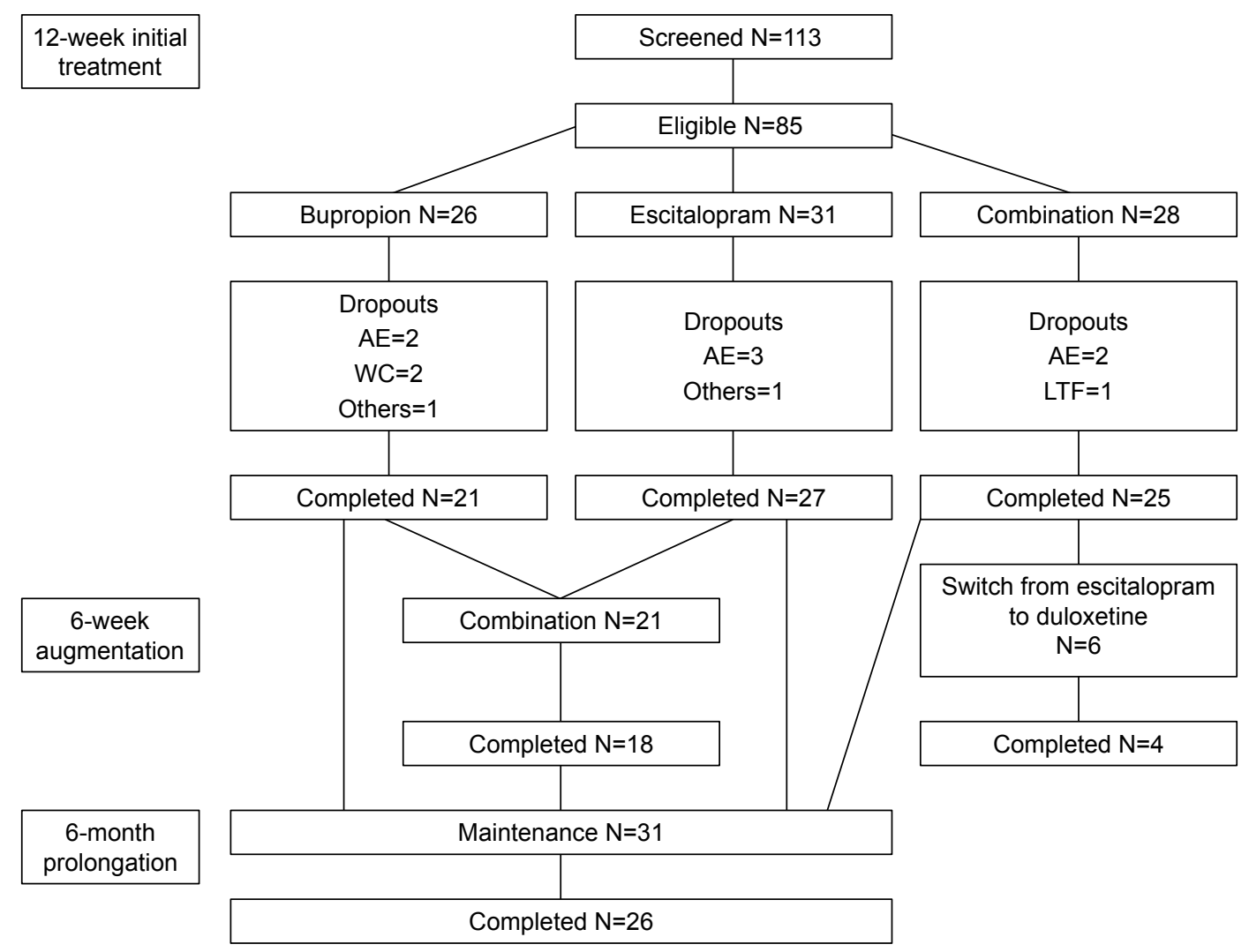

Figure I Patient enrollment at the Royal Institute of Mental Health Research in a double-blind, randomized trial of antidepressant monotherapy vs combination treatment, using escitalopram and bupropion in major depressive disorder patients.

Abbreviations: AE, adverse effect; LTF, lost to follow-up; WC, withdrawn consent.

monotherapy, $13 \%$ in escitalopram monotherapy, and $11 \%$ in the combination treatment patients in the first 12 weeks.

Following the initial 12-week treatment period, 27 nonremitters participated in the 6-week augmentation (Figure 1). Of these patients, 11 came from bupropion monotherapy, 10 from escitalopram monotherapy, and 6 from combination treatment. Since the monotherapy non-remitters received the same combination of escitalopram and bupropion in the augmentation phase, they were analyzed as one group.

\section{Primary outcome - initial treatment}

Hypothesis 1. To investigate the speed of onset of efficacy and remission, the overall HAM-D17 scores were analyzed first. Post-hoc LSD pairwise comparisons showed that in terms of the mean response at week 1 , combination treatment was significantly more beneficial relative to bupropion monotherapy (Figure 2). Remission rate comparison showed that the week 2 remission rate of combination treatment was significantly higher than of both monotherapies (Figure 3). On average, remitters had lower baseline HAM-D17 scores than non-remitters, $19.9 \pm 4.2$ vs $22.0 \pm 4.7$, respectively ( $\mathrm{F}(1$, 79) $=4.03, P=0.048)$.

Hypothesis 2. HAM-D17 final remission rate comparisons showed that combination treatment had a significantly higher week 12 remission rate than bupropion monotherapy, 15 remitters vs 7 , respectively $\left(\chi^{2}(1)=3.97, P=0.043\right.$;

Table I Patient demographics at the Royal Institute of Mental Health Research in a double-blind randomized trial of antidepressant monotherapy vs combination treatment, using escitalopram and bupropion in major depressive disorder patients

\begin{tabular}{l|l|l|l}
\hline & Escitalopram & Bupropion & Combination \\
\hline Age, mean (SD) & $40.6(13.0)$ & $40.8(10.8)$ & $38.1(10.3)$ \\
Sex (F/M), n (\% female) & $20 / 11$ (65) & $12 / 14(46)$ & $19 / 10(66)$ \\
Marital status* (Mar/N), n (\% Mar) & $15 / 16(48)$ & $14 / 12(54)$ & $16 / 12(57)$ \\
\hline
\end{tabular}

Notes: *Married includes married and living with someone as if married; Nonmarried includes divorced, widowed, separated, never married. There was no statistical difference in ages $\left(P>0.05\right.$, using one-way analysis of variance), sex distribution $\left(c^{2}=3.6 \mathrm{I}, P=0.16\right)$, and marital status $\left(c^{2}=0.90, P=0.64\right)$.

Abbreviations: $\mathrm{F}$, female; $\mathrm{M}$, male; Mar, married; $\mathrm{N}$, nonmarried. 


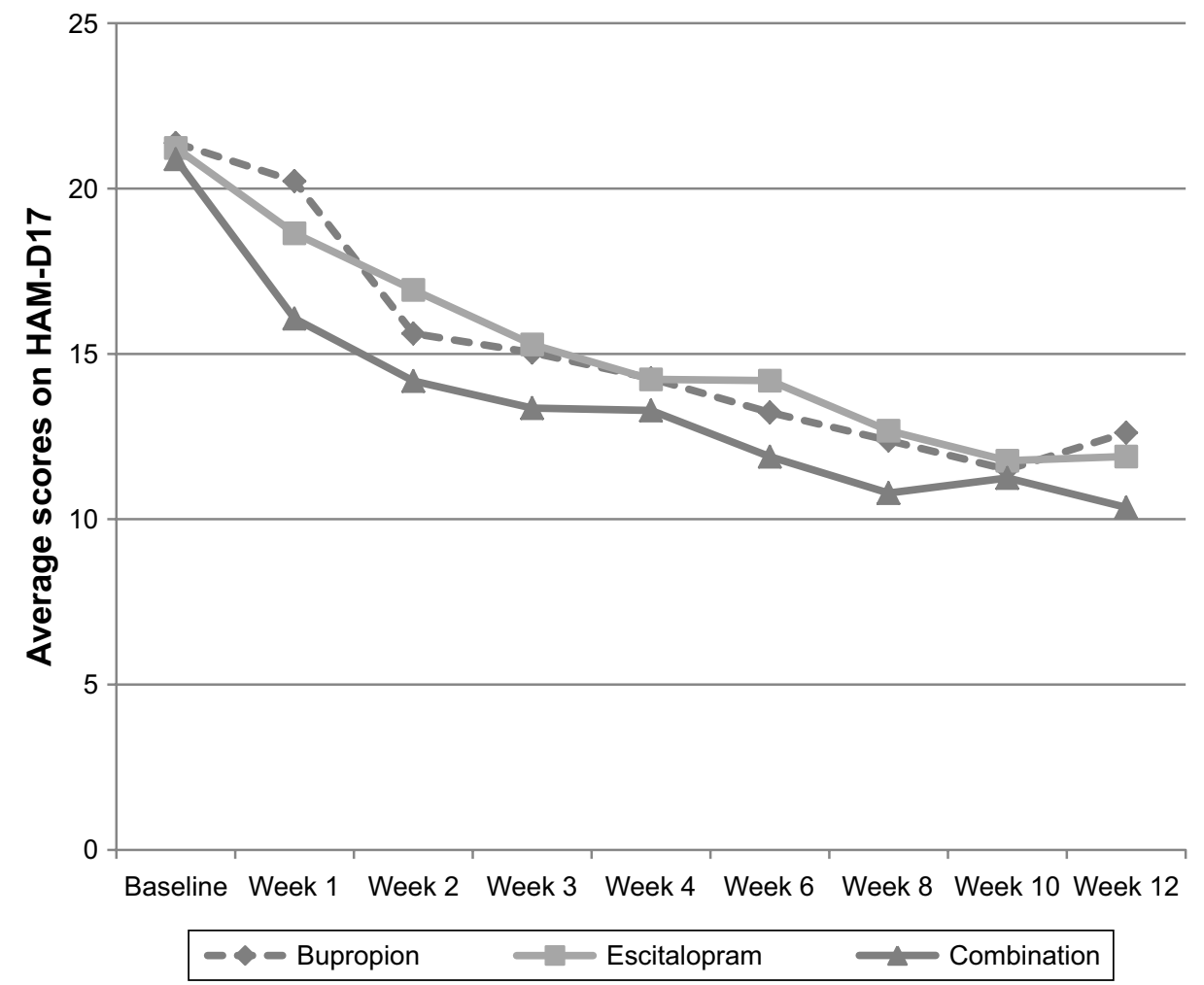

Figure 2 Mean scores on the HAM-DI7 (0-42) by week, for all major depressive disorder patients (last observation carried forward) during 12 weeks of initial treatment at the Royal Institute of Mental Health Research in a double-blind, randomized trial of antidepressant monotherapy vs combination treatment, using escitalopram and bupropion.

Note: Statistically significant difference existed in week I between the combination treatment and bupropion monotherapy in antidepressant response (LSD, $P=0.022$ ). Abbreviations: HAM-D 17, 17-item Hamilton Rating Scale for Depression; LSD, least significant difference.

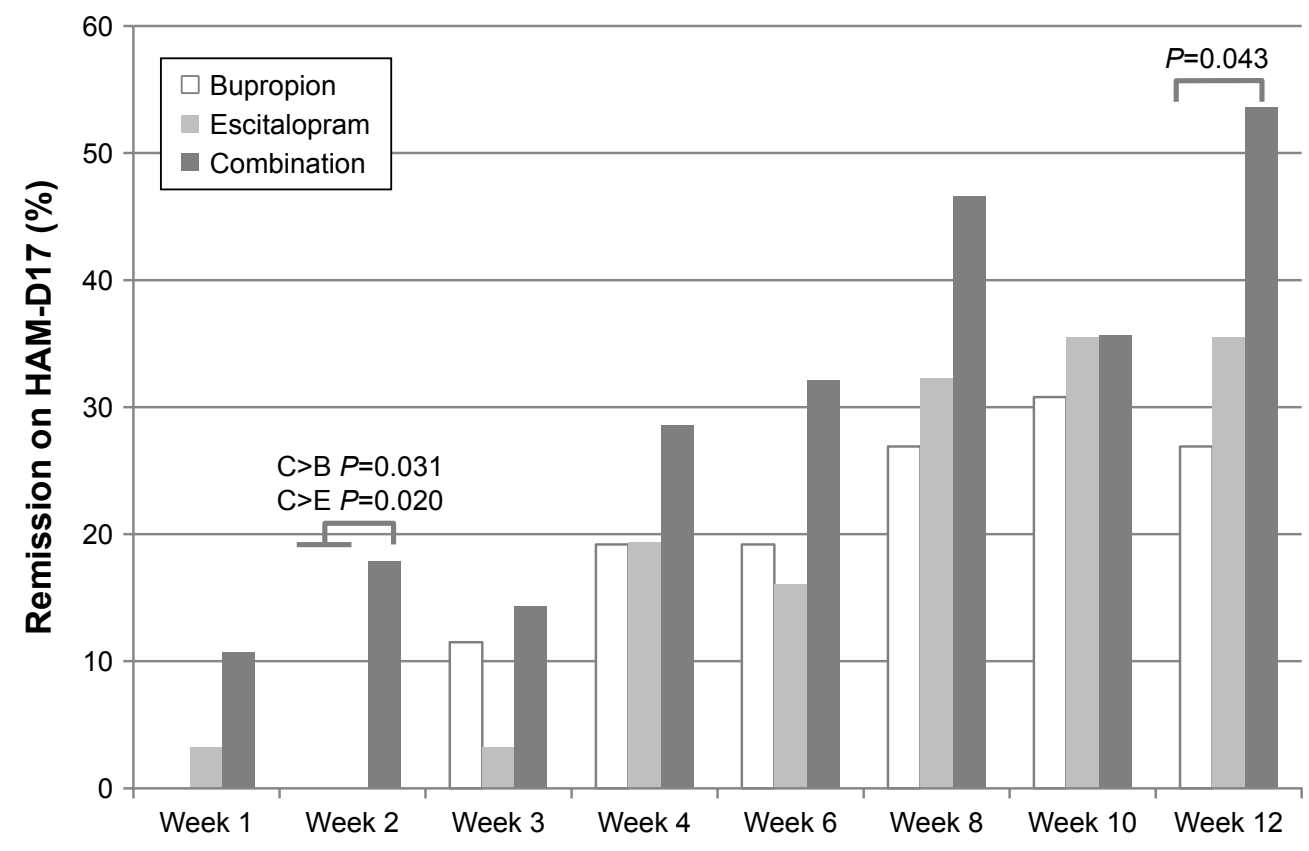

Figure 3 Percentage of remission on the HAM-DI7 ( $\leq 7$ ) by week, for all major depressive disorder patients (last observation carried forward) during 12 weeks of initial treatment at the Royal Institute of Mental Health Research in a double-blind randomized trial of antidepressant monotherapy vs combination treatment, using escitalopram and bupropion.

Note: Statistically significant differences existed in week 2 between the combination arm and both monotherapies, ie, 5 vs 0 on bupropion (Fisher's Exact test, $P=0.031$ ) and 0 on escitalopram (Fisher's Exact test, $P=0.020$ ).

Abbreviation: HAM-D 17, 17-item Hamilton Rating Scale for Depression. 
Figure 3). Combination week 12 remission was only numerically superior to escitalopram monotherapy $(P=0.16)$, which produced 11 final remitters.

\section{Secondary outcome - initial treatment}

Hypothesis 1 . The analysis of overall MADRS scores showed no significant effect of treatment $(P=0.55)$. MADRS remission rate comparisons confirmed the week 2 superiority of combination treatment compared with both monotherapies (combination vs escitalopram, Fisher's exact test, $P=0.003$, and combination vs bupropion, $\chi^{2}(1)=4.78, P=0.029$; see Figure 4). Remitters showed no baseline difference from non-remitters on the MADRS $(P=0.10)$.

Hypothesis 2. The MADRS week 12 remission rates showed no significant difference between treatments (Figure 4).

\section{Primary outcome - augmentation in non-remitters}

The 6-week augmentation trial lead to a significant response on the HAM-D17 in initial HAM-D17 non-remitters, which differed between the escitalopram and bupropion combination group and the combination non-remitters, in which escitalopram was switched for duloxetine $(F(1,25)=4.84$, $P=0.037$; Table 2).

Hypothesis 3. The augmentation HAM-D17 remission rates were $7 / 21$ in escitalopram and bupropion combination treatment and $0 / 6$ in the switch for duloxetine group $(P=0.13$; Figure 5).

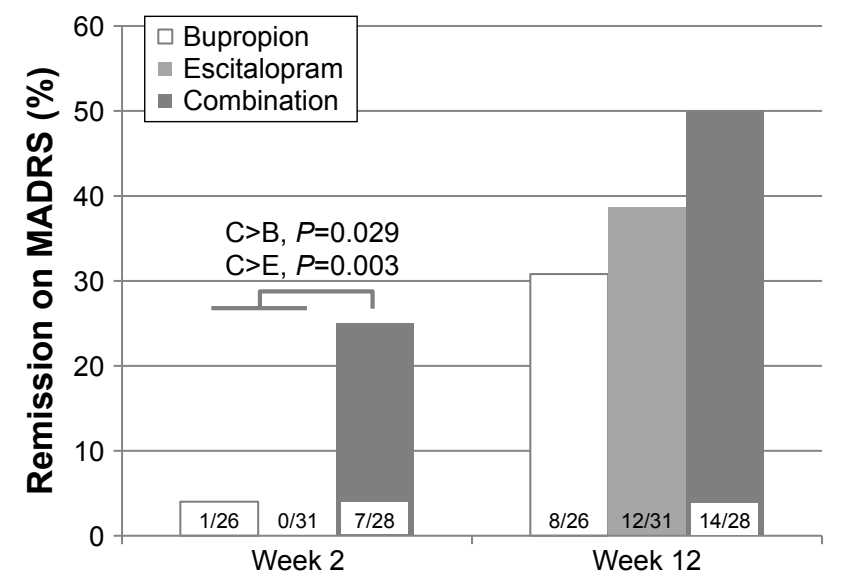

Figure 4 Percentage of remission on the MADRS $(\leq 10)$ by week, for all major depressive disorder patients (last observation carried forward) during 12 weeks of initial treatment at the Royal Institute of Mental Health Research in a double-blind randomized trial of antidepressant monotherapy vs combination treatment, using escitalopram and bupropion.

Note: The week 12 remission rate of the combination treatment on MADRS was not significantly different from escitalopram or bupropion monotherapies $(P=0.38$ and $P=0.15$, respectively).

Abbreviation: MADRS, Montgomery-Asberg Depression Rating Scale.
Table 2 Mean scores on the HAM-DI7 (0-42) in a doubleblind, 6-week augmentation trial in escitalopram or bupropion monotherapy non-remitters who received augmentation with the other drug, and in escitalopram and bupropion combination non-remitters who switched escitalopram for duloxetine (last observation carried forward)

\begin{tabular}{l|l|l|l}
\hline & Combination & $\begin{array}{l}\text { Duloxetine } \\
\text { switch }\end{array}$ & Significance \\
\hline $\mathrm{N}$ & $2 \mathrm{I}$ & 6 & $\mathrm{~ns}$ \\
Baseline & $14.3 \pm 5 . \mathrm{I}$ & $18.7 \pm 7.3$ & $\mathrm{~F}(\mathrm{I}, 25)=4.84, \mathrm{P}=0.037$ \\
Week 6 & $12.0 \pm 8.1$ & $19.8 \pm 6.3$ & \\
\hline & Remitters & Non-remitters & \\
\hline $\mathrm{N}$ & 7 & 20 & $\mathrm{~F}(\mathrm{I}, 24)=4.50, \mathrm{P}=0.044$ \\
Baseline & $10.9 \pm 4.8$ & $16.8 \pm 5.4$ & $\mathrm{~F}(\mathrm{I}, 24)=20.45, P<0.00 \mathrm{I}$ \\
Week 6 & $3.9 \pm 2.3$ & $17.2 \pm 6.6$ & $\mathrm{~L}$
\end{tabular}

Abbreviation: HAM-DI7, I7-item Hamilton Rating Scale for Depression.

\section{Secondary outcome - augmentation in non-remitters}

As the MADRS secondary outcome, the difference in mean scores of the combination group and the switch group showed significance at the end of the 6-week augmentation $(\mathrm{F}(1,24)=9.99, P=0.004)$. The mean scores of remitters vs non-remitters were significantly different at baseline as well as at week $6(\mathrm{~F}(1,24)=5.06, P=0.034$, and $\mathrm{F}(1,24)=30.14$ $P<0.001$, respectively).

Hypothesis 3. The augmentation MADRS remission rates were 6/21 in escitalopram and bupropion combination treatment and $0 / 6$ in the switch for duloxetine group $(P=0.28)$.

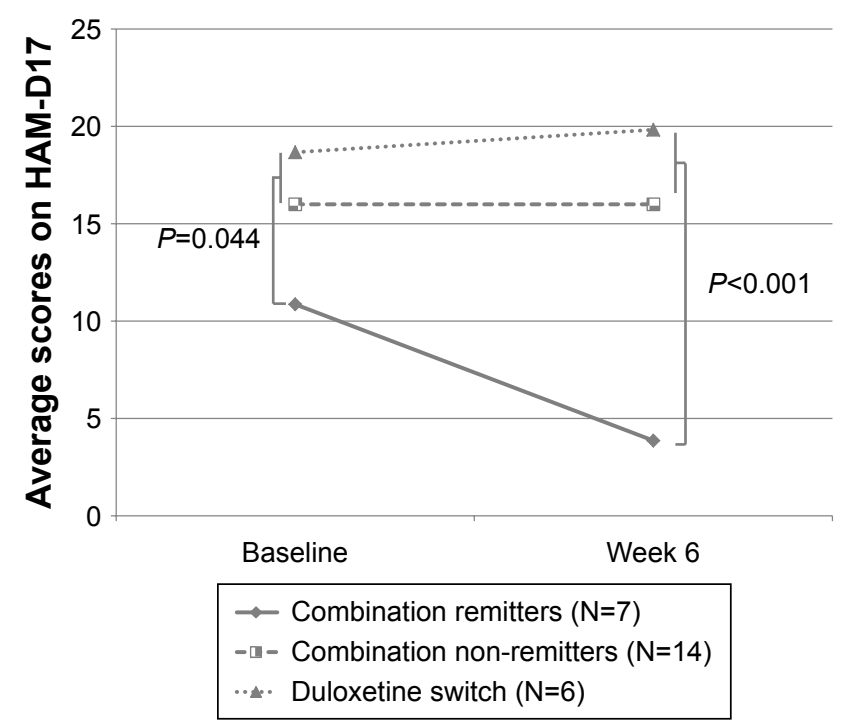

Figure 5 Mean scores on the HAM-DI7 (0-42) in a double-blind, 6-week augmentation trial in escitalopram or bupropion monotherapy non-remitters who received augmentation with the other drug, and in escitalopram and bupropion combination non-remitters who switched escitalopram for duloxetine (last observation carried forward).

Note: The mean score of remitters was lower than of non-remitters both at baseline and week $6(F(I, 24)=4.50, P=0.044$, and $F(I, 24)=20.45, P<0.00 I$, respectively). Abbreviation: HAM-D 17, I7-item Hamilton Rating Scale for Depression. 


\section{Dose regimen - augmentation phase}

The mean dose level of the added drug and that of the primary drug at the end of the 6-week augmentation were analyzed. There was no significant difference between treatment groups in the mean levels of the primary drug and the added drug ( $P=0.82$ and $P=0.91$, respectively) or between remitters and non-remitters $(P=0.33$ and $P=0.34$, respectively). The mean level of the primary drug was $3.8 \pm 0.4$ in patients coming from 12 -week bupropion monotherapy, $3.7 \pm 0.5$ in patients from 12 -week escitalopram monotherapy, and $3.7 \pm 0.8$ in patients from 12-week combination treatment; these levels represent bupropion, escitalopram and bupropion, respectively. The mean dose level of the augmentation drug was $2.7 \pm 1.4$ in patients coming from 12 -week bupropion monotherapy, $2.8 \pm 1.2$ in patients from 12 -week escitalopram monotherapy, and 3.0 \pm 0.9 in patients from 12 -week combination treatment; these levels correspond to escitalopram, bupropion, and duloxetine, respectively.

\section{Six-month prolongation in remitters}

The remitters participated in a 6 month prolongation in which response was assessed monthly on the HAM-D17. Some of the initial week 12 remitters and augmentation week 18 remitters
( $n=40)$ were lost to follow-up ( $n=9$ ) or dropped out during the prolongation $(n=5)$, but they were all in remission at the time of dropout. The 31 patients who participated in the prolongation were analyzed in an LOCF manner. The HAM-D17 month 6 remission rate was $90 \%$. Post-hoc LSD testing showed significant response differences between bupropion and combination at each visit throughout the prolongation after baseline, as well as a significant response difference between bupropion and escitalopram, except for month 2 (Figure 6; bupropion vs combination at month $1, P=0.006$; month $2, P=0.009$; month 3, $P=0.001$; month 4, $P=0.030$; month 5, $P=0.008$; month $6, P=0.003$; and bupropion vs escitalopram at month 1 , $P=0.046$; month 2, $P=0.12$; month $3, P=0.005$; month 4, $P=0.016$; month 5, $P=0.034$; and month 6, $P=0.001$ ).

Hypothesis 4. At month 6, 5/6 of bupropion remitters, $11 / 11$ of escitalopram remitters, and $12 / 14$ of combination remitters were still in remission. No significant difference in month 6 remission rates existed.

\section{Dose regimen - 6-month prolongation in remitters}

The mean dose level was $3.8 \pm 0.4$ in bupropion remitters, $3.7 \pm 0.6$ in escitalopram remitters, and $3.1 \pm 1.1$ in combination

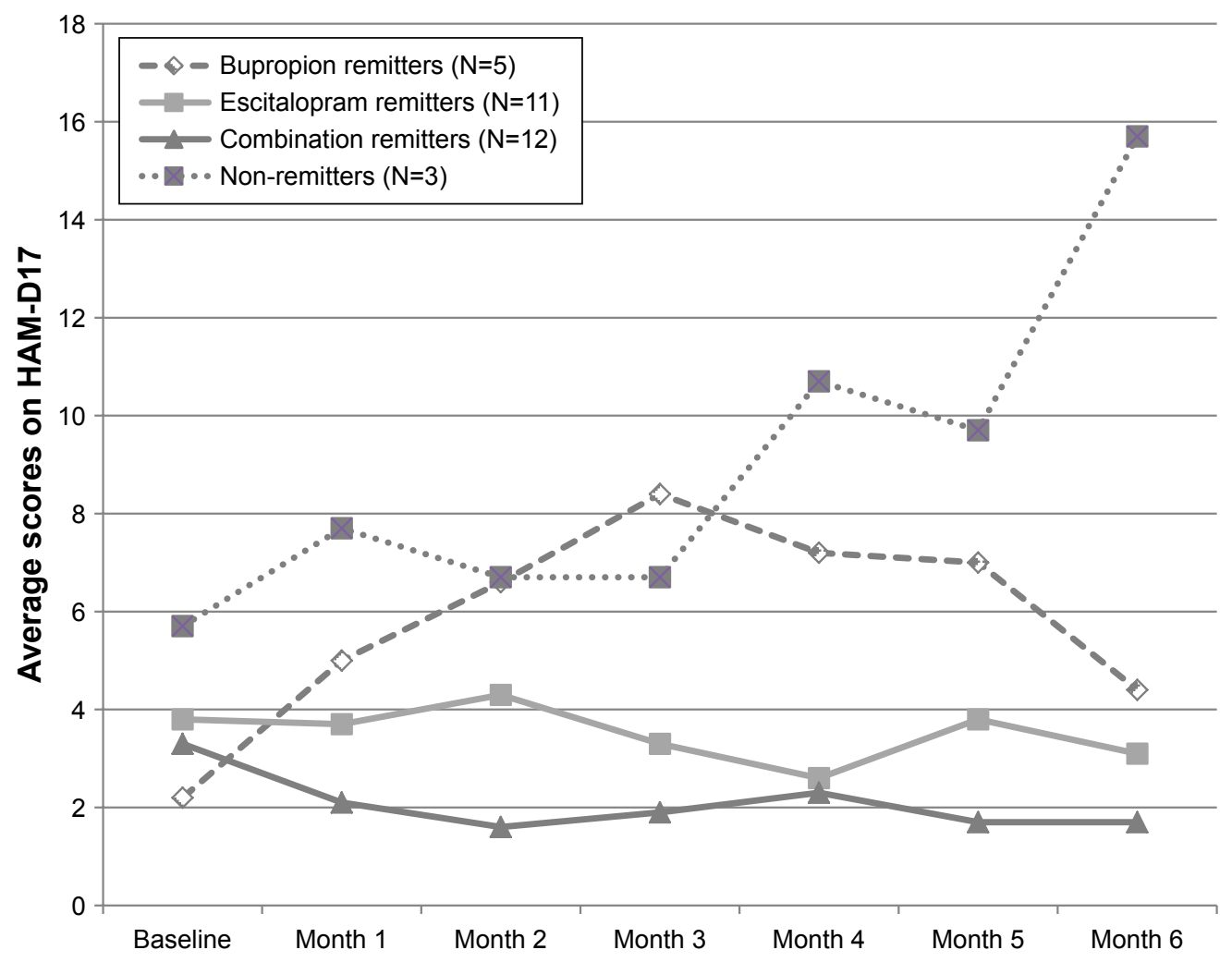

Figure 6 Mean scores on the HAM-DI7 (0-42) in a double-blind 6-month prolongation in week 12 initial treatment and week 6 augmentation remitters (last observation carried forward) on antidepressant monotherapy or combination treatment, using escitalopram and bupropion.

Abbreviation: HAM-D 17, 17-item Hamilton Rating Scale for Depression. 


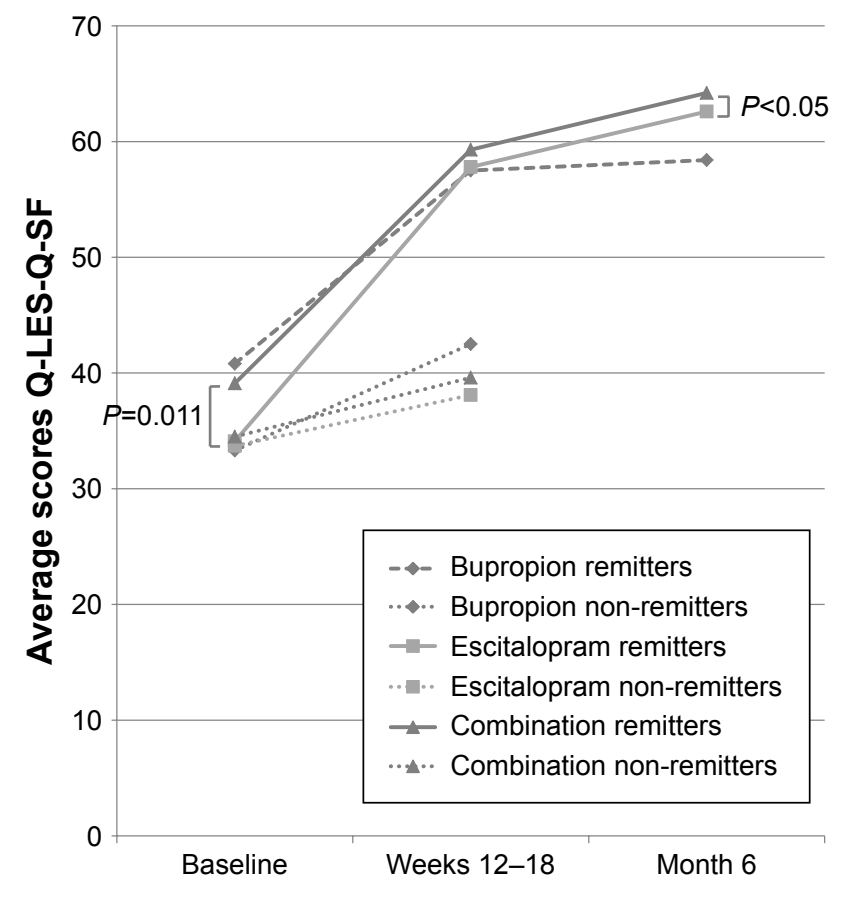

Figure 7 Mean scores on the Q-LES-Q-SF (0-80) for all patients (last observation carried forward) enrolled in a randomized, double-blind 12-week trial of antidepressant monotherapy vs combination treatment, including a double-blind 6-week augmentation in non-remitters, and a double-blind 6-month prolongation in both week 12 and week 18 remitters.

Abbreviation: Q-LES-Q-SF, Quality of Life Enjoyment and Satisfaction Questionnaire-short form.

remitters, without any significant difference between groups $(P=0.25)$ or remitters and non-remitters $(P=0.81)$.

\section{Analysis of self-assessment scale}

Post-hoc LSD pairwise comparisons showed that the combination treatment was overall significantly superior to escitalopram monotherapy at weeks $12-18$ on the selfassessment scale, the Quality of Life Enjoyment and Satisfaction Questionnaire-short form (Q-LES-Q-SF; $P=0.032$; Figure 7). Overall, remitters scored significantly higher than non-remitters both at baseline and week $12(\mathrm{~F}(1,78)=6.71$, $P=0.011$ and $\mathrm{F}(1,78)=71.49, P<0.001$, respectively). An analysis of only the remitters who participated in the 6-month prolongation, showed a significant difference between month 6 remitters and month 6 non-remitters at month 6 $(\mathrm{F}(1,26)=6.34, P=0.018)$. LSD testing showed a significantly higher mean score for combination treatment over escitalopram monotherapy at baseline $(P=0.043)$ and at month 6 $(P=0.022)$ between the 6-month prolongation patients.

\section{Discussion}

The present analysis of a subsample of MDD patients ( $\mathrm{n}=85$ ) was conducted within a larger trial $(n=245)$ that has been published and showed no final difference between escitalopram and bupropion combination from treatment initiation and either monotherapy. ${ }^{20}$ However, the larger, multicenter trial was subject to substantial methodological limitations. The same accounts for the multicenter-based CO-MED study, in which current combination strategy was investigated. In contrast to these larger trials holding methodological complications, multiple single-unit studies showed superior remission rates for a medicinal combination approach from treatment initiation in MDD.

A first justification for the investigation of current subsample was the strikingly lower dropout rate in this subsample compared with the other units within the larger trial, despite the use of higher dose regimens. Another reason justifying this subanalysis was the statistically significantly different patient presentation in the current sample, contrasting with those of the US sample. There were a significantly greater number of patients in this subsample meeting criteria for melancholia, whereas there were significantly more patients with comorbid anxiety in the NYC subsample. ${ }^{20}$ Nevertheless, in the overall trial, escitalopram was used at a mean dose that was higher than the maximum recommended one ( $20 \mathrm{mg} /$ day), and outperformed significantly bupropion. ${ }^{20}$

It is important to mention that none of the patients at baseline had an abnormally long corrected QT interval and that there were no cardiac complications linked to this parameter. These results are thus consistent with those of Wade et al who used doses of escitalopram as high as $50 \mathrm{mg} /$ day in patients with MDD without significant complications. ${ }^{22}$ Similar high doses of escitalopram in the use for obsessive compulsive disorder and binge eating achieved higher therapeutic benefits. ${ }^{23-26}$

The escitalopram-bupropion combination produced evidence for the more rapid onset of remission at week 2, using both the HAM-D17 and the MADRS due in part to no one achieving remission at this time point on a single medication in contrast to patients from other centers. As well in the overall sample of 245 patients, this difference was also statistically significant using the HAM-D29 scale which addresses a wider variety of symptoms than the HAM-D17 and the 10-item MADRS. ${ }^{20}$ It is noteworthy that even using the HAM-D17, there were more remitters in the combination group than in both monotherapy arms combined. It is important to note that at this time point in the study, the dose regimens were still within the label of both medications. Consequently, using two drugs from treatment initiation may be beneficial for some patients with MDD.

Throughout the subsequent 10 -week period, there was only numerical superiority of the combination approach over monotherapies, with the exception of the 12-week time period, when the combination approach statistically 
surpassed the bupropion monotherapy and resulted in a remission rate $>50 \%$. This specific combination did not produce clear evidence of superiority over escitalopram alone. It is nevertheless important to point out that doses of escitalopram were optimized beyond the current label of the drug (ie, 30 and $40 \mathrm{mg} /$ day).

In support of the benefits of the combination approach, the addition of the other drug in monotherapy non-remitters brought a third of patients below the remission threshold. In contrast, the combination non-remitters did not improve over the same 6-week period by switching escitalopram for duloxetine. These results obtained under double-blind conditions are thus fully consistent with the results of the STAR*D study - level 2, whereby bupropion addition to an ongoing regimen of the SSRI citalopram produced a $30 \%$ remission rate measured on the HAM-D17 scale, as in the present sample. ${ }^{5}$ The optimal dose of $120 \mathrm{mg}$ /day of duloxetine actively engaging the NE transporter was reached only in two of the six patients because of tolerability issues. ${ }^{27}$ Consequently, most patients in this group were not on a serotonin-norepinephrine reuptake inhibitor regimen of duloxetine.

In the prolongation phase, the remitters, independently of the drugs given, were all on optimized dose regimens and $90 \%$ maintained remission. In addition, their enhanced quality of life as measured by their self-assessment was also sustained. Therefore, the fourth hypothesis that the combination treatment would be superior to the monotherapies in sustaining remission could not be supported. These results are therefore in line with the clinical lore that the regimen of medications that gets patients well will keep them well.

Finally, different life circumstances between the current sample and the larger sample may have played a role in the differential outcome between the regions. Sociodemographic characteristics of income in a subsample of patients who had children between age 7 and 17, showing $63 \%$ of the NYC patients earned $<\$ 15 \mathrm{~K}$ in contrast to the $>\$ 40 \mathrm{~K}$ by $81 \%$ of the Ottawa region participants, likely reflected the significantly different unemployment rates observed in the larger sample, ie, $53 \%$ vs 35\%, respectively. ${ }^{20,28}$ The NYC patients enrolled in the larger sample may have presented with another depression type (ie, with anxious features), possibly attributable to more difficult life situations.

\section{Limitations}

The present analyses have, however, the following limitations. Patients were not stratified in the analysis for comorbid anxiety features vs MDD with high melancholia or without comorbidities. Therefore, the suggested relation between anxiety and the preferential responsiveness to escitalopram rather than bupropion or their combination could have been investigated more in depth. Secondly, the drug side effects were not compared between treatment groups in this subsample, although they have been published for the larger trial. ${ }^{20}$ However, the dropout rates between therapy arms were not significantly different and the sustained efficacy and low dropout during the 6-month prolongation confirmed that the use of higher dose regimens was well tolerated. Furthermore changes in dose levels were always implemented with the agreement of the patients.

\section{Conclusion}

In summary, the combination of escitalopram and bupropion led to a faster onset of remission in this patient group, assessed at week 2 using standard doses. This combination subsample analysis is consistent with results of the four prior double-blind studies showing remission rates of $50 \%$ using two medications with complementary mechanism of action from treatment initiation. ${ }^{15-18}$ This study also provided evidence for the strategy of augmentation when incomplete response is attained, as shown with other combinations of medications. ${ }^{29}$ Finally, evidence was provided for the effectiveness of maintaining optimized regimens of medications to sustain remission in MDD.

\section{Acknowledgments}

PB received a Canada Research Chair from the federal government, research grants and/or honoraria from Allergan, Biovail, Bristol Myers Squibb, Janssen, Lundbeck, Otsuka, Pierre Fabre Médicaments, Pfizer, and Sunovion.

\section{Disclosure}

Study medication and matching placebo were provided by Biovail and Lundbeck. The authors report no other conflicts of interest in this work.

\section{References}

1. Kessler C, Mcgonagle KA, Swartz M, Blazer DG, Nelson B. Sex and depression in the National Comorbidity Survey I: lifetime prevalence chronicity and recurrence. J Affect Disord. 1993;29:85-96.

2. World Health Organization. Depression [webpage on the Internet]. Geneva: World Health Organization; 2018 [Accessed May 3, 2018]. Available from: http://www.who.int/en/news-room/fact-sheets/detail/depression.

3. Smith D, Dempster C, Glanville J, Freemantle N, Anderson I. Efficacy and tolerability of venlafaxine compared with selective serotonin reuptake inhibitors and with selective serotonin reuptake inhibitors and other antidepressants: a meta-analysis. Br J Psychiatry. 2002;180: 396-404.

4. Thase ME, Haight BR, Richard N, et al. Remission rates following antidepressant therapy with bupropion or selective serotonin reuptake inhibitors: a meta-analysis of original data from 7 randomized controlled trials. J Clin Psychiatry. 2005;66(8):974-981. 
5. Trivedi MH, Rush AJ, Wisniewski SR, et al. Evaluation of outcomes with citalopram for depression using measurement-based care in STAR*D: implications for clinical practice. Am J Psychiatry. 2006; 163(1):28-40.

6. Fava M, Davidson KG. Definition and epidemiology of treatmentresistant depression. Psychiatr Clin North Am. 1996;19(2):179-200.

7. Nierenberg AA, Farabaugh AH, Alpert JE, et al. Timing of onset of antidepressant response with fluoxetine treatment. Am J Psychiatry. 2000;157(9):1423-1428.

8. Szegedi A, Jansen WT, van Willigenburg AP, van der Meulen E, Stassen HH, Thase ME. Early improvement in the first 2 weeks as a predictor of treatment outcome in patients with major depressive disorder: a meta-analysis including 6562 patients. J Clin Psychiatry. 2009;70(3):344-353.

9. Nemeroff CB. Prevalence and management of treatment-resistant depression. J Clin Psychiatry. 2007;68(8):17-25.

10. Blier P. Rational site-directed pharmacotherapy for major depressive disorder. Int J Neuropsychopharmacol. 2014;17(7):997-1008.

11. Kennedy SH, Lam RW, McIntyre RS, et al. Canadian Network for Mood and Anxiety Treatments (CANMAT) Clinical guidelines for the management of major depressive disorder in adults. Section 3 . Pharmacological treatments. Can J Psychaitry. 2016;61(9):540-560.

12. Cleare A, Pariante CM, Young AH, et al. Evidence-based guidelines for treating depressive disorders with antidepressants: a revision of the 2008 British Association for Psychopharmacology guidelines. J Psychopharmacol. 2015;29(5):459-525.

13. Keller M, Lavori P, Mueller T. Time to recovery, chronicity, and levels of psychopathology in MDD - 5-year follow-up. Arch Gen Psychiatry. 1992;49:809-816.

14. Oluboka OJ, Katzman MA, Habert J, et al. Functional recovery in major depressive disorder: providing early optimal treatment for the individual patient. Int J Neuropsychopharmacol. 2018;21(2):128-144.

15. Maes M, Libbrecht I, van Hunsel F, Campens D, Meltzer HY. Pindolol and mianserin augment the antidepressant activity of fluoxetine in hospitalized major depressed patients, including those with treatment resistance. J Clin Psychopharmacol. 1999;19(2):177-182.

16. Nelson JC, Mazure CM, Jatlow PI, Bowers MB, Price LH. Combining norepinephrine and serotonin reuptake inhibition mechanisms for treatment of depression: a double-blind, randomized study. Biol Psychiatry. 2004;55(3):296-300.

17. Blier P, Gobbi G, Turcotte JE, et al. Mirtazapine and paroxetine in major depression: a comparison of monotherapy versus their combination from treatment initiation. Eur Neuropsychopharmacol. 2009;19(7):457-465.
18. Blier P, Ward HE, Tremblay P, Laberge L, Hébert C, Bergeron R. Combination of antidepressant medications from treatment initiation for major depressive disorder: a double-blind randomized study. Am J Psychiatry. 2010;167(3):281-288.

19. Rush AJ, Trivedi MH, Stewart JW, et al. Combining Medications to Enhance Depression Outcomes (CO-MED): acute and long-term outcomes of a single-blind randomized study. Am J Psychiatry. 2011; 168(7):689-701.

20. Stewart JW, McGrath PJ, Blondeau C, et al. Combination antidepressant therapy for major depressive disorder: speed and probability of remission. J Psychiatr Res. 2014;52(1):7-14.

21. IBM Corp. IBM SPSS Statistics for Windows, Version 22.0. Armonk, NY: IBM Corp; Released 2013.

22. Wade AG, Crawford GM, Yellowlees A. Efficacy, safety and tolerability of escitalopram in doses up to $50 \mathrm{mg}$ in Major Depressive Disorder (MDD): an open-label, pilot study. BMC Psychiatry. 2011;11(1):42.

23. Guerdjikova AI, Mcelroy SL, Kotwal R, et al. High-dose escitalopram in the treatment of binge-eating disorder with obesity: a placebo-controlled monotherapy trial. Hum Psychopharmacol. 2008;23(1):1-11.

24. Rabinowitz I, Baruch Y, Barak Y. High-dose escitalopram for the treatment of obsessive-compulsive disorder. Int Clin Psychopharmacol. 2008; 23(1):49-53.

25. Dougherty DD, Jameson M, Deckersbach T, et al. Open-label study of high (30 mg) and moderate $(20 \mathrm{mg})$ dose escitalopram for the treatment of obsessive-compulsive disorder. Int Clin Psychopharmacol. 2009;24(6):306-311.

26. Shim G, Park HY, Jang JH, et al. What is the optimal dose of escitalopram for the treatment of obsessive-compulsive disorder? A naturalistic open-label study. Int Clin Psychopharmacol. 2011;26(5):284-290.

27. Vincent S, Bieck PR, Garland EM, et al. Clinical assessment of norepinephrine transporter blockade through biochemical and pharmacological profiles. Circulation. 2004;109(25):3202-3207.

28. Batten LA, Hernandez M, Pilowsky DJ, et al. Children of treatmentseeking depressed mothers: a comparison with the sequenced treatment alternatives to relieve depression (STAR*D) child study. J Am Acad Child Adolesc Psychiatry. 2012;51(11):1185-1196.

29. Henssler J, Bschor T, Baethge C. Combining antidepressants in acute treatment of depression: a meta-analysis of 38 studies including 4511 patients. Can J Psychiatry. 2016;61(1):29-43.
Neuropsychiatric Disease and Treatment

\section{Publish your work in this journal}

Neuropsychiatric Disease and Treatment is an international, peerreviewed journal of clinical therapeutics and pharmacology focusing on concise rapid reporting of clinical or pre-clinical studies on a range of neuropsychiatric and neurological disorders. This journal is indexed on PubMed Central, the 'PsycINFO' database and CAS,

\section{Dovepress}

and is the official journal of The International Neuropsychiatric Association (INA). The manuscript management system is completely online and includes a very quick and fair peer-review system, which is all easy to use. Visit http://www.dovepress.com/testimonials.php to read real quotes from published authors. 